\title{
Whole body magnetic resonance in indolent lymphomas under watchful waiting: The time is now
}

\author{
Massimo Galia ${ }^{1}$ - Domenico Albano ${ }^{1}$ (D) Corrado Tarella $^{2}$ - Caterina Patti $^{3}$. \\ Luca Maria Sconfienza ${ }^{4,5}$ - Antonino Mulè ${ }^{3}$ Pierpaolo Alongi ${ }^{6}$ - Massimo Midiri ${ }^{1}$. \\ Roberto Lagalla ${ }^{1}$
}

Received: 29 April 2017 /Revised: 26 July 2017 / Accepted: 11 September 2017 /Published online: 10 October 2017

(C) European Society of Radiology 2017

\begin{abstract}
The indolent non-Hodgkin lymphomas (i-NHLs) are characterised by 'indolent' clinical behaviour with slow growth and prolonged natural history. The watchful waiting (WW) strategy is a frequently employed treatment option in these patients. This implies a strict monitoring by imaging examinations, including $18 \mathrm{~F}$ fluorodeoxyglucose-positron emission tomography/computed tomography (18F-FDG-PET/CT) and CT. A major concern is radiation exposure due to regularly monitoring by conventional imaging procedures. Several studies have demonstrated the reliability of whole-body magnetic resonance imaging (WB-MRI) for lymphoma staging. WB-MRI could be useful for active surveillance in iNHLs providing the suspect of disease progression that can be then confirmed by additional diagnostic procedures, including 18F-FDG-PET/CT. The directive $2013 / 59$ by the European Union claims that if a radiation-free imaging technique allows obtaining the same diagnostic results, it should be invariably used.
\end{abstract}

Domenico Albano

albanodomenico@me.com

1 Department of Radiology, Di.Bi.Med., University of Palermo, Via del Vespro 127, 90127 Palermo, Italy

2 Hemato-Oncology Division, European Institute of Oncology, Via Giuseppe Ripamonti 435, 20141 Milan, Italy

3 Department of Hematology I, Azienda Ospedaliera Ospedali Riuniti Villa Sofia-Cervello, Viale Trabucco 180, 90146 Palermo, Italy

4 Unit of Diagnostic and Interventional Radiology, IRCCS Istituto Ortopedico Galeazzi, Via Riccardo Galeazzi 4, 20161 Milano, Italy

5 Department of Biomedical Sciences for Health, Università degli Studi di Milano, Via Festa del Perdono 7, 20122 Milano, Italy

6 Department of Radiological Sciences, Nuclear Medicine Unit, Fondazione Istituto G. Giglio, Contrada Pietrapollastra-Pisciotto, 90015 Cefalù, Italy
In this setting, WB-MRI may be considered a reasonable option in i-NHLs under WW, replacing imaging modalities that cause exposure to ionising radiations. This will help to reduce the cancer risk in i-NHL patients for whom chemo-/radiotherapy remain the usual treatment options following the usually long WW phase. The scientific community should raise the awareness of the risk of ionising radiations in i-NHLs and the emphasise the need for establishing the proper place of WB-MRI in lymphoma imaging. Key Points

- Watchful waiting is a reasonable option in patients with indolent non-Hodgkin lymphomas.

- Imaging is crucial to monitor patients with indolent nonHodgkin lymphomas.

- CT and ${ }^{18} F-F D G-P E T / C T$ are commonly used, implying a substantial radiation exposure.

- WB-MRI is highly reliable in lymphoma staging.

- WB-MRI may be considered to monitor indolent nonHodgkin lymphomas under watchful waiting.

Keywords Magnetic resonance imaging - Whole body imaging · Ionising radiation · Lymphoma · Watchful waiting

$\begin{array}{ll}\text { Abbreviations } & \\ { }^{18} \text { F-FDG-PET/ } & { }^{18} \text { F-fluorodeoxyglucose-positron emission } \\ \text { CT } & \text { tomography/computed tomography } \\ \text { FL } & \text { Follicular lymphoma } \\ \text { i-NHL } & \text { Indolent non-Hodgkin's lymphoma } \\ \text { LL } & \text { Lymphoplasmacytic lymphoma } \\ \text { MCL } & \text { Mantle cell lymphomas } \\ \text { MZL } & \text { Marginal zone lymphoma } \\ \text { SLL } & \text { Small lymphocytic lymphoma } \\ \text { WB-MRI } & \text { Whole body magnetic resonance imaging } \\ \text { WW } & \text { Watchful waiting }\end{array}$




\section{Introduction}

Indolent non-Hodgkin's lymphomas (i-NHLs) are mainly characterised by 'indolent' clinical behaviour with slow growth, prolonged natural history, and lack of clinical symptoms [1]. Patients with i-NHLs are often actively monitored using blood tests, clinical examination, and imaging follow- up, rather than immediately treated. This watchful waiting (WW) strategy enables therapies to be delayed until they are really needed. Armitage and Longo suggested that asymptomatic clinical picture, no lymphomatous involvement in dangerous sites, and patient's desire to delay the treatment are the key factors that should encourage the clinician to consider WW as a treatment option [2].
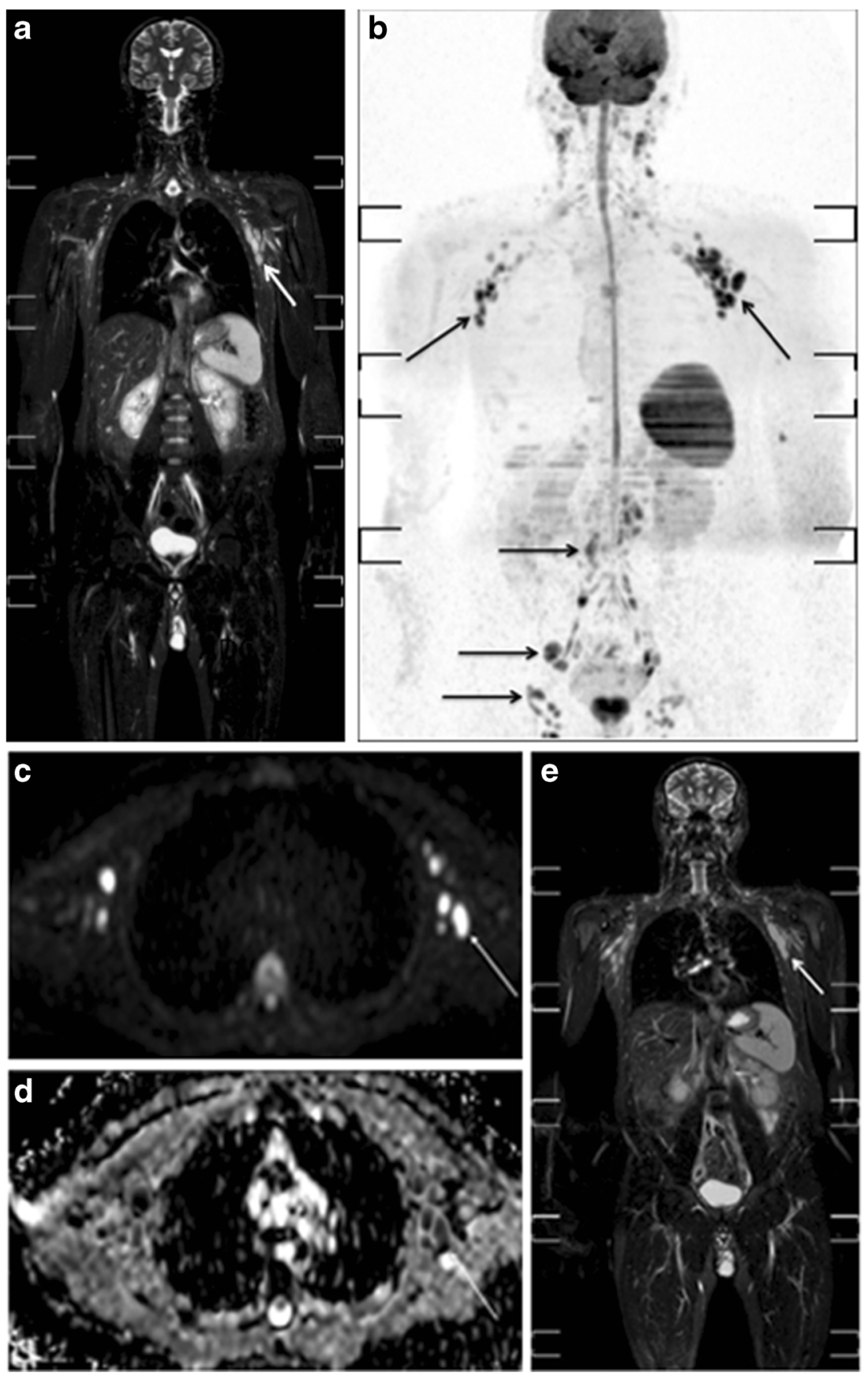

Fig. 1 A 50-year-old man with non-Hodgkin's follicular lymphoma. At the time of diagnosis, coronal T2w-STIR (a), MIP grey-scale inverted diffusion-weighted imaging (DWI) (b), axial high b-value DWI (c) and ADC map (d) showed multiple pathological lymph nodes (arrows) in supradiaphragmatic and subdiaphragmatic regions. After 6 months under watchful waiting, coronal T2w-STIR (e), MIP grey-scale inverted DWI (f), axial high b-value DWI (g) and ADC map (h) showed a slight increase of lymph nodes (arrows), especially in the left axillary region. The patient was asymptomatic with a low tumour burden and is still under watchful waiting 


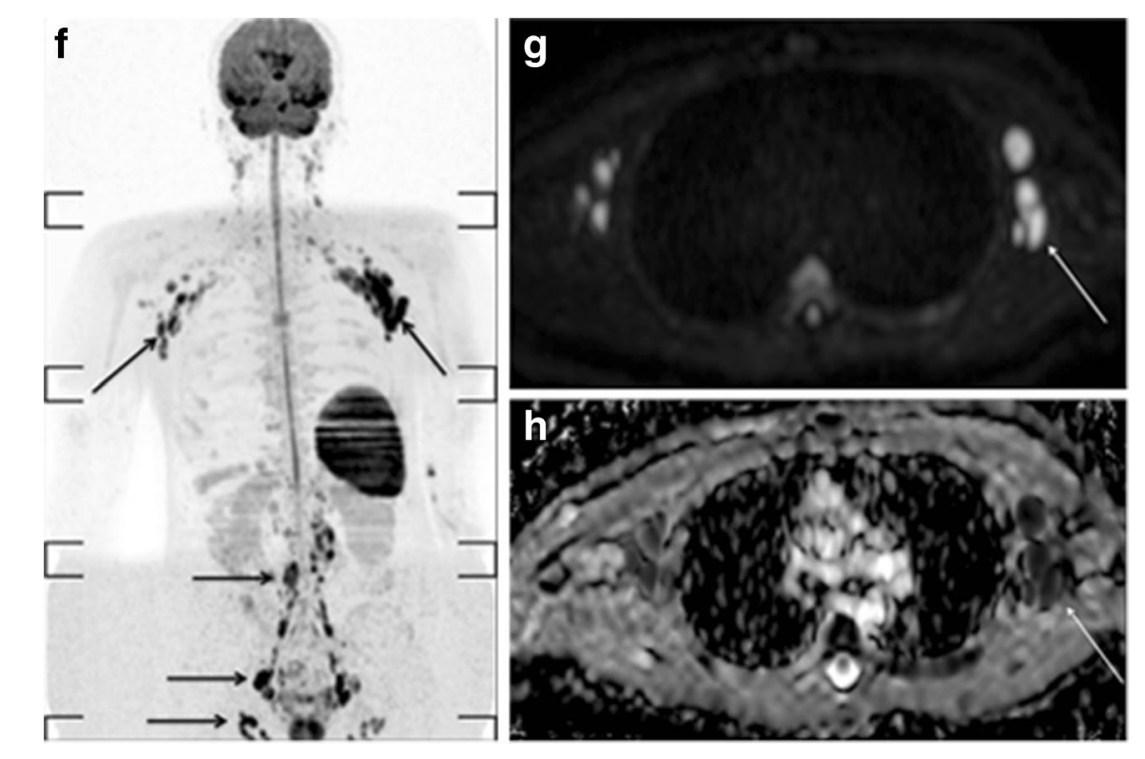

Fig. 1 continued.

The most common i-NHL subtypes include follicular lymphoma (FL), marginal zone lymphoma (MZL), lymphoplasmacytic lymphoma (LL), small lymphocytic lymphoma (SLL), and a small subset of mantle cell lymphoma (MCL). FL is the second most common NHL following diffuse large-B-cell lymphoma, representing more than $20 \%$ of NHLs. Taken together, all i-NHLs have a high incidence, close to that of the most frequent diffuse large-B-cell lymphoma subtype [2].

\section{The watchful waiting (WW) strategy in indolent non-Hodgkin's lymphoma (i-NHL)}

Previous studies have not demonstrated an increased overall survival rate with active treatment over WW in asymptomatic patients with low-grade FL, making WW a reasonable treatment approach in the immunochemotherapy era [3]. The Italian Society of Haematology, the Italian Society of Experimental Haematology, and the Italian Bone Marrow Transplantation Group have published guidelines for the management of both follicular and non-follicular i-NHL patients $[4,5]$, suggesting WW as a valuable initial option especially in asymptomatic patients with FL stage II-IV and low tumour burden [4]. Similar to FL, asymptomatic patients with splenic MZL are commonly monitored without any treatment since WW does not compromise survival [6]; nevertheless, patients should be treated if they are symptomatic or have bulky disease or cytopenia [7]. Some MALT lymphoma patients can be monitored with WW as well, without significantly modifying survival rates [8]. The WW strategy can also be pursued with good results in some ocular, adnexal, and pulmonary MALT lymphomas $[9,10]$. The LL patients may be treated with several approaches, including WW in those who are asymptomatic with low tumour burden [11]. In patients with SLL, a WW strategy is indicated as an initial approach, with treatment considered only for patients with disease-related symptoms or progressive or advanced stage disease [12]. A small subset of MCL, identified by lack of SOX11 expression, is associated with indolent behaviour and favorable prognosis with no need for immediate treatment, compared to the common SOX11+MCL [13]. These patients are generally asymptomatic, with a normal serum lactate dehydrogenase level and no lymphadenopathies. Again, these patients may be monitored through periodical clinical and radiological examinations, even delaying treatment for several years [14].

\section{Imaging monitoring and WW}

Imaging evaluation is crucial for monitoring patients with iNHL under WW, for surveillance purposes of their variable risk of disease progression. ${ }^{18} \mathrm{~F}$-fluorodeoxyglucose-positron emission tomography/computed tomography $\left({ }^{18} \mathrm{~F}\right.$-FDG-PET/ $\mathrm{CT}$ ) is the standard imaging modality for FDG-avid lymphoma staging, whereas CT is recommended for non-FDG-avid histotypes and is commonly used for monitoring and imaging follow-up [15]. Thus, ${ }^{18} \mathrm{~F}$-FDG-PET/CT is widely applied in FL for staging and end-of-treatment assessment, while it is not recommended in other i-NHL subtypes. Ultrasound is also used to follow i-NHLs as a cheap, safe, and effective diagnostic tool that does not involve ionising radiations. However, ultrasound may be less reproducible compared to other imaging modalities, it has reduced accuracy on retroperitoneum evaluation due to possible overlaying fat or bowel gas, and it is unable to investigate either the mediastinum or the bones. In addition, it is noteworthy that novel and effective, though very expensive, drugs such as immunomodulatory agents and 
checkpoint inhibitors, are under development for patients with lymphoma. Despite the documented clinical efficacy, conventional imaging evaluation procedures are often inadequate for clearly detecting disease during treatment with these novel targeted drugs [16]. Indeed, continued revision of response criteria, along with a definition of precise imaging timepoints, is urged [16]. Thus, guidelines on imaging examinations should be updated for the monitoring of i-NHL patients undergoing both WW and treatment with novel targeted drugs.

A major concern regarding the follow-up of lymphoma patients through CT is the possible association of several scans with increased cancer risk. Brenner and Hall argued that up to $2 \%$ of cancers can be related to radiation exposure associated with CT examinations [17]. Previous studies reported an increased secondary cancer risk due to surveillance $\mathrm{CT}$ scans in patients with NHL $[18,19]$. As regards to the use of ${ }^{18}$ F-FDG$\mathrm{PET} / \mathrm{CT}$, it is well known that involves exposure to a substantial dose of ionising radiations, again with increasing cancer risk [20]. However, ${ }^{18}$ F-FDG-PET/CT has a crucial role in FL management. ${ }^{18} \mathrm{~F}$-FDG-PET/CT is highly sensitive for disease staging in FL, allowing better identification of nodal and extra-nodal disease locations in comparison with CT [21].
Fig. 2 A 37-year-old man with non-Hodgkin's follicular lymphoma. At the time of diagnosis, coronal T1w (a), axial high bvalue diffusion-weighted imaging (DWI) (b) and ADC map (c) showed a pathological mesenteric lymph node (arrow). After 6 months under watchful waiting, the lymph node did not show any change in size as demonstrated by coronal T1w (d), axial high bvalue DWI (e) and ADC map (f)
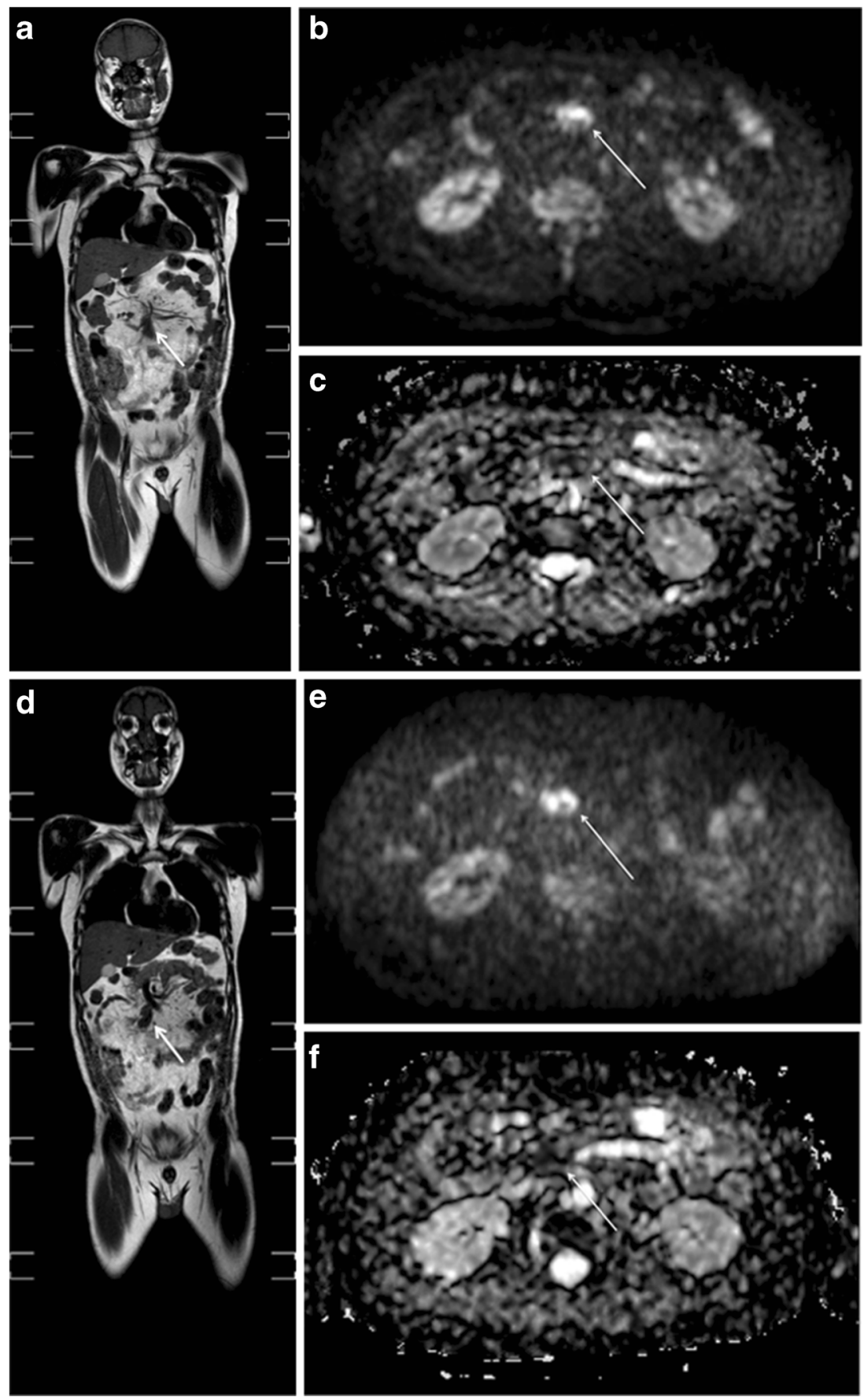
Moreover, it enables accurate detection of lymphoma transformation due to the significantly increased FDG uptake in histologically transformed areas [22]. Furthermore, it has to be stressed that, among all the i-NHL subtypes, FL has a higher risk of secondary tumours or transformation in a more aggressive lymphoma, most commonly diffuse large B-cell lymphoma [23].

\section{The emerging role of whole-body magnetic resonance imaging for disease monitoring in $\mathrm{NHL}$}

Over the last years, several studies have demonstrated the good reliability of whole-body magnetic resonance imaging (WB-MRI) for lymphoma staging, mainly using ${ }^{18} \mathrm{~F}-\mathrm{FDG}-$ $\mathrm{PET} / \mathrm{CT}$ as the reference standard [24-27]. WB-MRI enables avoiding radiation exposure and intravenous contrast agent administration, allowing the evaluation of all potential lymphomatous sites through morphological and functional sequences (Figs. 1 and 2) [28]. Indeed, while CT provides only morphological information regarding nodal and extra-nodal locations of disease, diffusion-weighted imaging allows the random motion of water molecules in biological structures to be investigated and allows easy identification of hypercellular tissues like lymphoma [29]. In addition, several studies have shown that WB-MRI equals or betters CT in lymphoma staging $[30,31]$, suggesting it be used in place of CT. Both WBMRI and ${ }^{18} \mathrm{~F}$-FDG-PET/CT have been shown to be reliable tools for the detection of bone marrow involvement in patients with lymphoma [32, 33]. In this setting, both techniques seem to have distinct diagnostic accuracy in aggressive lymphomas, showing reduced sensitivity for the detection of low-volume bone marrow involvement in i-NHLs, where imaging modalities are complementary to bone marrow biopsy [32, 33]. In fact, bone marrow biopsy is still recommended in NHL [15], being essential for both diagnosis and evaluation of marrow cellularity and hematopoietic reserve [34-36], while it is no longer required for Hodgkin's lymphoma [15]. Finally, previous studies have shown the potential role of WB-MRI in the early detection of osteonecrotic lesions in lymphoma patients treated with high doses of corticosteroids, allowing prompt referral of these patients to orthopaedists and providing the opportunity for early intervention [37-39].

Of note, the directive 2013/59 by the European Union claims that if a radiation-free imaging technique enables the same diagnostic results to be obtained, it should always be used [40]. Based on this recommendation, WB-MRI should be considered as a reasonable option in i-NHLs under WW. When possible, WB-MRI may replace those imaging modalities that involve exposure to ionising radiations, allowing the cancer risk in patients who will ultimately receive chemo- and radiotherapy following the usually long WW phase to be reduced. Although WB-MRI has demonstrated similar diagnostic accuracy to ${ }^{18} \mathrm{~F}$-FDG-PET/CT in aggressive lymphomas, the latter imaging modality is well and widely standardized, and WB-MRI will probably have a complementary role in these patients.

However, WB-MRI could be an ideal diagnostic tool in iNHLs, allowing regular disease monitoring and providing early detection of signs of disease progression or transformation. These warning signs may be the occurrence of new nodal or extra-nodal lesions, progressive and rapid enlargement of pathological lymph nodes with short doubling time of the maximum diameter, bulky disease (greater diameter $\geq 7 \mathrm{~cm}$ ) and serosal effusions [5]. These findings identified by WBMRI may then be validated by additional ${ }^{18} \mathrm{~F}$-FDG-PET/CT, the capability of which in identifying the histological transformation of FL is well established [22]. Moreover, Wu et al. have recently found that FL and diffuse large B-cell lymphoma could be differentiated by means of texture analysis on post-contrast T1-weighted images [41]. It might potentially help in early identification of a transformed lymphoma during imaging follow-up of FL patients, but this needs to be confirmed by larger studies.

\section{Perspectives}

In conclusion, i-NHLs are lymphomas characterised by 'indolent' behaviour, with slow growth, and a prolonged natural history. WW is a valuable and widely employed option to manage i-NHL patients with a low tumour burden. In this setting, patients need to be regularly monitored through imaging examinations, including $\mathrm{CT}$ and ${ }^{18} \mathrm{~F}$-FDG-PET/CT, with exposure to substantial ionising radiations. Since WB-MRI has a well-demonstrated reliability in lymphoma patients, future studies should focus on the application of this imaging modality in the follow-up of patients with i-NHL under WW in order to reduce radiation exposure and, as consequence, secondary cancer risk. The scientific community should raise the awareness of the risk of ionising radiation, defining the proper place of WB-MRI in lymphoma imaging and establishing precise imaging timepoints for the optimal management of patients with i-NHL, with particular attention to those under a WW strategy.

Funding The authors state that this work has not received any funding.

\section{Compliance with ethical standards}

Guarantor The scientific guarantor of this publication is Massimo Galia.

Conflict of interest The authors of this manuscript declare no relationships with any companies whose products or services may be related to the subject matter of the article. 
Statistics and biometry No complex statistical methods were necessary for this article.

Ethical approval Institutional review board approval was not required because this article does not involve patient data.

Methodology Editorial

\section{References}

1. Lunning M, Vose JM (2012) Management of indolent lymphoma: Where are we now and where are we going. Blood Rev 26:279-288

2. Armitage JO, Longo DL (2016) Is watch and wait still acceptable for patients with low-grade follicular lymphoma? Blood 127:2804 2808

3. Sorigue M, Sancho JM, Ribera JM (2016) Open questions in watchful waiting for follicular lymphoma. Br J Haematol (in press)

4. Zinzani PL, Marchetti M, Billio A et al (2013) Expert Panel of the Italian Society of Hematology. SIE, SIES, GITMO revised guidelines for the management of follicular lymphoma. Am J Hematol 88:185-192

5. Tarella C, Arcaini L, Baldini L et al (2015) Italian Society of Hematology, Italian Society of Experimental Hematology, and Italian Group for Bone Marrow Transplantation guidelines for the management of indolent, nonfollicular B-cell lymphoma (marginal zone, lymphoplasmacytic, and small lymphocytic lymphoma). Clin Lymphoma Myeloma Leuk 15:75-85

6. Arcaini L, Lazzarino M, Colombo N et al (2006) Splenic marginal zone lymphoma: a prognostic model for clinical use. Blood 107: 4643-4649

7. Matutes E, Oscier D, Montalban C et al (2008) Splenic marginal zone lymphoma proposals for a revision of diagnostic, staging and therapeutic criteria. Leukemia 22:487-495

8. Raderer M, Kiesewetter B, Ferreri AJ (2016) Clinicopathologic characteristics and treatment of marginal zone lymphoma of mucosa-associated lymphoid tissue (MALT lymphoma). CA Cancer J Clin 66:153-171

9. Tanimoto K, Kaneko A, Suzuki S et al (2006) Long-term follow-up results of no initial therapy for ocular adnexal MALT lymphoma. Ann Oncol 17:135-140

10. Troch M, Streubel B, Petkov V, Turetschek K, Chott A, Raderer M (2007) Does MALT lymphoma of the lung require immediate treatment? An analysis of 11 untreated cases with long-term follow-up. Anticancer Res 27:3633-3637

11. Ardeshna KM, Smith P, Norton A et al (2003) Long-term effect of a watch and wait policy versus immediate systemic treatment for asymptomatic advanced-stage non-Hodgkin lymphoma: a randomised controlled trial. Lancet 362:516-522

12. Tadmor T, Polliack A (2012) Optimal management of older patients with chronic lymphocytic leukemia: some facts and principles guiding therapeutic choices. Blood Rev 26:15-23

13. Martin P, Chadburn A, Christos P et al (2009) Outcome of deferred initial therapy in mantle-cell lymphoma. J Clin Oncol 27:12091213

14. Dreyling M, Ferrero S, Hermine O (2014) How to manage mantle cell lymphoma. Leukemia 28:2117-2130

15. Cheson BD, Fisher RI, Barrington SF et al (2014) Recommendations for initial evaluation, staging and response assessment of Hodgkin and non-Hodgkin lymphoma: the lugano classification. J Clin Oncol 32:3059-3067

16. Cheson BD, Ansell S, Schwartz L et al (2016) Refinement of the Lugano classification response criteria for lymphoma in the era of immunomodulatory therapy. Blood 128:2489-2496
17. Brenner DJ, Hall EJ (2007) Computed tomography: an increasing source of radiation exposure. N Engl J Med 357:2277-2284

18. Shenoy P, Sinha R, Tumeh JW, Lechowicz MJ, Flowers CR (2010) Surveillance computed tomography scans for patients with lymphoma: is the risk worth the benefits? Clin. Lymphoma Myeloma Leuk 10:270-277

19. Chien SH, Liu CJ, Hu YW et al (2015) Frequency of surveillance computed tomography in non-Hodgkin lymphoma and the risk of secondary primary malignancies: a nationwide population-based study. Int J Cancer 137:658-665

20. Huang B, Law MW, Khong PL (2009) Whole-body PET/CT scanning: estimation ofradiation dose and cancer risk. Radiology 251: 166-174

21. Luminari S, Biasoli I, Arcaini L et al (2013) The use of FDG-PET in the initial staging of 142 patients with follicular lymphoma: a retrospective study from the FOLL05 randomized trial of the Fondazione Italiana Linfomi. Ann Oncol 24:2108-2112

22. Smith SD, Redman M, Dunleavy K (2015) FDG PET-CT in follicular lymphoma: a case-based evidence review. Blood 125:1078 1082

23. Lossos IS, Gascoyne RD (2011) Transformation of follicular lymphoma. Best Pract Res Clin Haematol 24:147-163

24. Albano D, Patti C, La Grutta L et al (2016) Comparison between whole-body MRI with diffusion-weighted imaging and PET/CT in staging newly diagnosed FDG-avid lymphomas. Eur J Radiol 85: 313-318

25. Mayerhoefer ME, Karanikas G, Kletter K et al (2015) Evaluation of diffusion-weighted magnetic resonance imaging for follow-up and treatment response assessment of lymphoma: results of an $18 \mathrm{~F}$ FDG-PET/CT-controlled prospective study in 64 patients. Clin Cancer Res 21:2506-2513

26. Lin C, Luciani A, Itti E et al (2010) Whole-body diffusion-weighted magnetic resonance imaging with apparent diffusion coefficient mapping for staging patients with diffuse large B-cell lymphoma. Eur Radiol 20:2027-2038

27. Littooij AS, Kwee TC, Barber I et al (2014) Whole-body MRI for initial staging of paediatric lymphoma: prospective comparison to an FDG-PET/CT-based reference standard. Eur Radiol 24:11531165

28. Albano D, La Grutta L, Grassedonio E et al (2016) Pitfalls in whole body MRI with diffusion weighted imaging performed on patients with lymphoma: what radiologists should know. Magn Reson Imaging 34:922-931

29. King AD, Ahuja AT, Yeung DK et al (2007) Malignant cervical lymphadenopathy: Diagnostic accuracy of diffusion-weighted MR imaging. Radiology 245:806-813

30. Kwee TC, Vermoolen MA, Akkerman EA et al (2013) Whole-body MRI, including diffusion-weighted imaging, for staging lymphoma: Comparison with $\mathrm{CT}$ in a prospective multicenter study. J Magn Reson Imaging 40:26-36

31. Balbo-Mussetto A, Cirillo S, Bruna R et al (2016) Whole-body MRI with diffusion-weighted imaging: A valuable alternative to contrast-enhanced CT for initial staging of aggressive lymphoma. Clinical Radiology 71:271-279

32. Adams HJ, Kwee TC, Vermoolen MA et al (2013) Whole-body MRI for the detection of bone marrow involvement in lymphoma: prospective study in 116 patients and comparison with FDG-PET. Eur Radiol 23:2271-2278

33. Albano D, Patti C, Lagalla R, Midiri M, Galia M (2017) Wholebody MRI, FDG-PET/CT, and bone marrow biopsy, for the assessment of bone marrow involvement in patients with newly diagnosed lymphoma. J Magn Reson Imaging 45:1082-1089

34. Iannitto E, Tripodo C (2011) How I diagnose and treat splenic lymphomas. Blood 117:2585-2595 
35. Vose JM (2015) Mantle cell lymphoma: 2015 update on diagnosis, risk stratification, and clinical management. Am J Hematol 90:739745

36. Kridel R, Mottok A, Farinha P et al (2015) Cell of origin of transformed follicular lymphoma. Blood 126:2118-2127

37. Albano D, Patti C, La Grutta L et al (2017) Osteonecrosis detected by whole body magnetic resonance in patients with Hodgkin lymphoma treated by BEACOPP. Eur Radiol 27:2129-2136

38. Littooij AS, Kwee TC, Goya E et al (2017) Whole-body MRI reveals high incidence of osteonecrosis in children treated for Hodgkin lymphoma. Br J Haematol 176:637-642
39. Albano D, Patti C, Sconfienza LM, Galia M (2017) Whole-body MRI in the early detection of multifocal osteonecrosis. Br J Radiol 190:20170240

40. Messina C, Banfi G, Aliprandi A et al (2016) Ultrasound guidance to perform intra-articular injection of gadolinium-based contrast material for magnetic resonance arthrography as an alternative to fluoroscopy: the time is now. Eur Radiol 26:1221-1225

41. Wu X, Sikiö M, Pertovaara H et al (2016) Differentiation of diffuse large B-cell lymphoma from follicular lymphoma using texture analysis on conventional MR images at 3.0 Tesla. Acad Radiol 23:696-703 